\title{
The effects of sociodemographic factors on quality of life among people aged 50 years or older are not unequivocal: comparing SF-12, WHOQOL-BREF, and WHOQOL-OLD
}

This article was published in the following Dove Medical Press journal: Clinical Interventions in Aging

\section{Robbert JJ Gobbens ${ }^{1-3}$ Roy Remmen ${ }^{3}$}

'Faculty of Health, Sports and Social Work, Inholland University of Applied Sciences, Amsterdam, the Netherlands; ${ }^{2}$ Zonnehuisgroep Amstelland, Amstelveen, the Netherlands; ${ }^{3}$ Department of Primary and Interdisciplinary Care, Faculty of Medicine and Health Sciences, University of Antwerp, Antwerp, Belgium
Correspondence: Robbert J] Gobbens Faculty of Health, Sports and Social Work, Inholland University of Applied Sciences, De Boelelaan II09, I08I HV Amsterdam, the Netherlands Tel +3 I 62 III 5578

Email robbert.gobbens@inholland.nl

\begin{abstract}
Objective: The effects of sociodemographic factors on quality of life in older people differ strongly, possibly due to the fact that different measurement instruments have been used. The main aim of this cross-sectional study is to compare the associations of sex, age, marital status, education, and income with quality of life assessed with the Short-Form Health Survey (SF-12), the World Health Organization Quality of Life Questionnaire-BREF (WHOQOL-BREF), and the World Health Organization Quality of Life Questionnaire-Older Adults Module (WHOQOL-OLD).

Methods: The associations between sociodemographic factors and eleven quality of life domains were examined using a sample of 1,492 Dutch people aged $\geq 50$ years. Participants completed the "Senioren Barometer", a web-based questionnaire including sociodemographic factors, the SF-12, the WHOQOL-BREF, and the WHOQOL-OLD.
\end{abstract}

Results: All the sociodemographic factors together explained a significant part of the variance of all the quality of life domains' scores, ranging from $5 \%$ to $17 \%$ for the WHOQOL-BREF, $5.8 \%$ to $6.7 \%$ for the SF-12, and $1.4 \%$ to $26 \%$ for the WHOQOL-OLD. Being a woman and being older were negatively associated with two and four quality of life domains, respectively. Being a woman, being married or cohabiting, and having higher education and a higher income were positively associated with six, six, one, and eleven quality of life domains, respectively. Conclusion: Our study showed that the associations of sociodemographic factors and quality of life in middle-aged and older people depend on the instruments used to assess quality of life. We recommend that health care and welfare professionals focus particularly on people with a low income and carry out interventions aimed at improving their quality of life.

Keywords: quality of life, older people, sociodemographic factors, SF-12, WHOQOL-BREF, WHOQOL-OLD

\section{Introduction}

Quality of life has been defined by the World Health Organization Quality of Life Group (1995, p. 1405) as "an individual's perception of their position in life in the context of the culture and value system in which they live and in relation to their goals, expectations, standards and concerns". ${ }^{1}$ The quality of life of older adults can be maintained at a high level, even with poor physical health, as long as they are satisfied with other domains of quality of life, such as the psychological, social relations, and environmental domains. ${ }^{2}$ For older adults, being in good health, feeling good, being active, having social relationships, helping other people, and living in a nice house in 
a good neighborhood (eg, availability of facilities and safety) are the most important factors for a good quality of life. ${ }^{3}$

Many studies have been conducted to assess the associations between sociodemographic factors (sex, age, marital status, education, and income) and quality of life in older adults..$^{4-9}$ However, no consensus exists on the effects of these factors on the quality of life of this target group. In relation to sex, Bilgili and Arpaci $^{10}$ found that men had higher scores on five of the six subscales of the World Health Organization Quality of Life Questionnaire-Older Adults Module (WHOQOL-OLD); they experienced a better quality of life than women. In the study by Lee et al, ${ }^{4}$ the quality of life of men was also higher than that of women. However, other studies have demonstrated no differences in quality of life between the two sexes, for example Top et al, ${ }^{8}$ Wiggins et al, ${ }^{11}$ and Chen et al. ${ }^{12}$

Younger age has been related to poorer quality of life in rural southern Brazil $;{ }^{13}$ Brazilian people in the age category 60-69 years rated their quality of life, assessed with the World Health Organization Quality of Life QuestionnaireBREF (WHOQOL-BREF), lower in the psychological and social relations domains and for overall quality of life than people over 80 years of age. ${ }^{6}$ However, these findings have not been supported by studies conducted in Europe; for example, Borglin et a ${ }^{14}$ reported lower quality of life in the oldest Swedish people and Soósová7 found the same among Slovak older people.

Soósová7 showed that living without a partner is negatively associated with quality of life. Bilgili and Arpaci ${ }^{10}$ also found higher scores on quality of life among older people living in a marital relationship; these people rated their quality of life higher in three domains of the WHOQOL-OLD (past, present, and future activities; social participation; and death and dying). On the other hand, using the same instrument, Gobbens and Van Assen ${ }^{15}$ demonstrated that marital status (married or cohabiting) was only associated with the quality of life domain intimacy, after controlling for other sociodemographic factors (age, education, and income), multimorbidity, and multidimensional frailty. Lee et $\mathrm{al}^{4}$ also showed that quality of life was higher for older people living with a spouse than for older people with no spouse; in this study, quality of life was assessed with the Short-Form Health Survey (SF-12). ${ }^{16}$

More years of education have been related to higher scores in psychological, social relations, and environmental quality of life. ${ }^{13}$ In addition, in a study by Gobbens et al, ${ }^{9}$ higher education was only significantly associated with better psychological and environmental quality of life. Baernholdt et al ${ }^{17}$ showed that social functioning was higher in older American people with a high school education or higher. Hilleras et $\mathrm{al}^{18}$ found no significant associations between educational level and quality of life in people aged 90 years or above. Soósová 7 observed better quality of life among higher-educated older people in the autonomy domain of the WHOQOL-OLD, but they experienced lower quality of life in the death and dying domain.

Chen et $\mathrm{al}^{12}$ showed that low income was related to poor quality of life in older people living alone in China, although they found no differences in quality of life between medium-high and high economic-level groups. In addition, Gambin et $\mathrm{al}^{13}$ showed that older Brazilian people with lower income were more likely to assess their quality of life as being lower, measured with both the WHOQOL-BREF and the WHOQOL-OLD. Gobbens et $\mathrm{al}^{9}$ found significant associations between higher income and higher quality of life in Dutch older people, after controlling for other sociodemographic variables and physical, psychological, as well as social frailty. On the contrary, Soósová 7 and Hilleras et a ${ }^{18}$ found no effect of income on quality of life. This can be explained by the inclusion of specific populations in both studies: people aged 60 years and older in the Košice region in Slovakia ${ }^{7}$ and people aged 90 years and older living in the inner part of Stockholm in Sweden. ${ }^{18}$

According to Hambleton et al, ${ }^{19}$ quality of life has been conceptualized and measured in a variety of ways, depending, for example, on the discipline, paradigm, target group, and time frame of the study examining quality of life. Over the years, several instruments have been developed for assessing quality of life in adults, for example, the SF-12 16 and the WHOQOL-BREF. ${ }^{20}$ Moreover, instruments have been developed in particular for assessing quality of life in older people, such as the World Health Organization Quality of Life-AGE questionnaire (WHOQOL-AGE), ${ }^{21}$ the Elderly Quality of Life Index, ${ }^{22}$ the WHOQOL-OLD, ${ }^{23}$ and the Older People's Quality of Life (OPQOL) questionnaire. ${ }^{24}$

Thus, in brief, a number of significant associations have been found with measures of quality of life, and the effects of sociodemographic factors on quality of life in older people appear to differ strongly. Factors that might interfere are the differences in the socioeconomic level of the various populations referred to in the literature, or their belonging to different cultures. Moreover, this may also be caused by the fact that different instruments have been used for measuring quality of life. Therefore, the main aim of the present crosssectional study is to compare the effects of sex, age, marital status, education, and income on quality of life assessed with the SF-12, the WHOQOL-BREF, and the WHOQOL-OLD. 
We decided to use these three instruments to measure quality of life in older adults, because these scales differ considerably from each other in content and have been validated extensively and used frequently for this age group. Furthermore, the SF-12, the WHOQOL-BREF, and the WHOQOL-OLD have recently been used in Dutch studies. ${ }^{15,25,26}$ Our second aim is to determine the associations between aforementioned sociodemographic factors and quality of life.

\section{Methods}

\section{Study population and data collection}

We used the "Senioren Barometer" to collect the data. This web-based questionnaire has been developed by the Academic Center Policy for the Elderly and Informal Care (Tranzo, Tilburg University, the Netherlands) aiming to assess the opinion of a panel of Dutch middle-aged and older people (aged 50 years and older) about different aspects of life. Older people can volunteer to take part, and participation is always without obligation. ${ }^{9,27}$

In the period December 2009 to January 2010, 1,942 people aged 50 years and older completed the "Senioren Barometer", including questions about sociodemographic factors, the SF-12, the WHOQOL-BREF, and the WHOQOLOLD. We excluded 450 cases from further analyses due to one or more missing values for sociodemographic factors and quality of life, yielding a sample size of 1,492.

\section{Measures}

\section{Sociodemographic factors}

The following sociodemographic factors were considered: age (in years), sex, marital status (five categories), education (five categories), and net income (nine categories). See Table 1 for a detailed description of the answering categories.

\section{The WHOQOL-BREF}

The WHOQOL-BREF was developed as a shortened version of the WHOQOL-100. ${ }^{20}$ This self-report questionnaire contains 24 items, which are categorized into four domains: physical health ( 7 items), psychological (6 items), social relations ( 3 items), and environmental ( 8 items). All these items were rated on a 5-point scale, with higher scores indicating better quality of life. The quality of life domain scores were calculated as usual by multiplying the mean domain score by a factor of 4 , resulting in a range from 4 to 20 for each domain. Many studies have shown that the WHOQOL-BREF has good psychometric properties for assessing quality of life in people aged 50 years and older. ${ }^{28,29}$ In the present study, the reliability expressed by the Cronbach's alpha for the physical,
Table I Descriptive statistics for participant characteristics $(n=I, 492)$

\begin{tabular}{|c|c|}
\hline Characteristic & n (\%) \\
\hline Age, mean $\pm S D$, range & $69.5 \pm 7.8,50-95$ \\
\hline \multicolumn{2}{|l|}{ Sex } \\
\hline Men & $900(60.3)$ \\
\hline Women & $592(39.7)$ \\
\hline \multicolumn{2}{|l|}{ Marital status } \\
\hline Married or cohabiting & $\mathrm{I}, 055(70.7)$ \\
\hline Single & $154(10.3)$ \\
\hline Divorced & $89(6.0)$ \\
\hline Widowed & I7| (II.5) \\
\hline Living apart together & $23(1.5)$ \\
\hline \multicolumn{2}{|l|}{ Education } \\
\hline None & $92(6.2)$ \\
\hline Primary & $161(10.8)$ \\
\hline Secondary & $663(44.4)$ \\
\hline $\begin{array}{l}\text { Polytechnics and higher } \\
\text { vocational training }\end{array}$ & $448(30.0)$ \\
\hline University & $128(8.6)$ \\
\hline \multicolumn{2}{|l|}{ Net monthly income* } \\
\hline$€ 999$ or less & $38(2.6)$ \\
\hline$€ I, 000-€ I, 499$ & $202(14.0)$ \\
\hline$€ I, 500-€ I, 999$ & $254(17.7)$ \\
\hline$€ 2,000-€ 2,499$ & $307(2 \mid .3)$ \\
\hline$€ 2,500-€ 2,999$ & $174(12.1)$ \\
\hline$€ 3,000-€ 3,499$ & $15 \mathrm{I}(10.5)$ \\
\hline$€ 3,500-€ 3,999$ & $81(5.6)$ \\
\hline$€ 4,000-€ 4,499$ & $56(3.9)$ \\
\hline$€ 4,500$ or more & $47(3.3)$ \\
\hline \multicolumn{2}{|l|}{ SF-12, mean $\pm S D$, range } \\
\hline Physical & $71.4 \pm 24.4,0-100$ \\
\hline Mental & $75.7 \pm 18.5,7.5-100$ \\
\hline \multicolumn{2}{|l|}{ WHOQOL-BREF, mean $\pm S D$, range } \\
\hline Physical health & $15.5 \pm 2.7,6.9-20$ \\
\hline Psychological & $15.1 \pm 2.1,7.3-20$ \\
\hline Social relations & $14.2 \pm 2.6,4-20$ \\
\hline Environmental & $15.9 \pm 2.2,8-20$ \\
\hline \multicolumn{2}{|l|}{ WHOQOL-OLD, mean $\pm S D$, range } \\
\hline Sensory abilities & $16.6 \pm 2.9,4-20$ \\
\hline Autonomy & $15.0 \pm 2.2,6-20$ \\
\hline Past, present, and future activities & $15.2 \pm 2.2,5-20$ \\
\hline Social participation & $15.5 \pm 2.6,4-20$ \\
\hline Death and dying & $15.1 \pm 3.2,4-20$ \\
\hline Intimacy & $14.4 \pm 3.1,4-20$ \\
\hline
\end{tabular}

Note: $* 182$ missing values.

Abbreviations: SF-12, Short-Form Health Survey; WHOQOL-BREF, World Health Organization Quality of Life Questionnaire-BREF; WHOQOL-OLD, World Health Organization Quality of Life Questionnaire-Older Adults Module. 
psychological, social relations, and environmental domains was $0.85,0.77,0.61$, and 0.80 , respectively.

\section{The SF-12}

The SF-12 is a widely used instrument for assessing quality of life. ${ }^{16}$ The SF-12 is derived from the SF- $36^{30}$ and contains only 12 items. These items are categorized into two domains, a physical and a mental domain, both including six items; the scores of the quality of life domains range from 0 to 100 , with higher scores referring to higher quality of life. Several studies have reported the good psychometric properties of the SF-12 in different age groups, including older persons, and a variety of countries. ${ }^{31,32}$ In the current study, the Cronbach's alpha was 0.86 and 0.79 for the physical and the mental domain of the SF-12, respectively.

\section{The WHOQOL-OLD}

The WHOQOL-OLD, just like the WHOQOL-BREF, was developed from the parent instrument, the WHOQOL-100. ${ }^{23}$ This questionnaire comprises 24 items belonging to six domains: sensory abilities, autonomy, past, present, and future activities, social participation, death and dying, and intimacy (4 items for each domain). The responses were rated on a 5-point scale (1-5). The domain score was calculated by summing the item scores, with a range from 4 to 20 ; higher scores equal better quality of life.

Many studies have reported the reliability and validity of the WHOQOL-OLD, ${ }^{33,34}$ including a study conducted in the Netherlands. ${ }^{35}$ In the present study, the reliability (Cronbach's alpha) for sensory abilities, autonomy, past, present, and future activities, social participation, death and dying, and intimacy was $0.87,0.67,0.70,0.81,0.83$, and 0.91 , respectively.

\section{Analysis strategies}

First, we determined the characteristics of the participants using descriptive statistics. Second, the associations between the five sociodemographic factors (age, sex, marital status, education, and income) and the domains of the SF-12, the WHOQOL-BREF, and the WHOQOL-OLD were examined. Eta was used to determine the associations between the 4 categorical variables (sex, marital status, education, and income) and the 12 quality of life domains. The associations between the continuous variable (age) and the quality of life domains were examined using Pearson's correlations.

Third, before we carried out multiple linear regression analyses with the quality of life domains of three scales as dependent variables and all five sociodemographic factors as independent variables, we created dummies for marital status
(" 1 " married or cohabiting and " 0 " otherwise) and sex (" 1 " woman and " 0 " man), as performed in a previous study. 9 We also incorporated the linear effects of age and education into these regression analyses. For income we used the interval midpoint of the answering categories (see Table 1), transformed the units to thousand euros, and considered this variable as quantitative. For each model related to the quality of life domains of the SF-12, the WHOQOL-BREF, and the WHOQOL-OLD, the explained variance $R^{2}$ was calculated.

Two-tailed tests were conducted; a $P$-value $<0.05$ was considered to be statistically significant. All the statistical analyses were carried out using the Statistical Package for Social Sciences (SPSS), version 22.0 (IBM Corporation, Armonk, NY, USA).

\section{Ethical considerations}

All procedures performed in our study were in accordance with ethical standards of the national research committee and with the 1964 Declaration of Helsinki and its later amendments or comparable ethical standards. Medical-ethical approval was not necessary, as particular treatments or interventions were not offered or withheld from respondents, this being the main criterion in medical-ethical procedures in the Netherlands according to the Medical Research Involving Subjects Act. ${ }^{36}$ Informed consent, in terms of giving information and maintaining confidentiality, was respected. In addition, return of the questionnaire was regarded as informed consent.

\section{Results}

\section{Participants' characteristics}

Of the 1,492 participants, $900(60.3 \%)$ were men. The mean age was 69.5 years (range 50-95 years, SD 7.8), and $70.0 \%$ were married or cohabiting. Most of the participants (44.4\%) had a level of education corresponding to secondary education, and $34.3 \%$ had an income $<€ 2,000$. Table 1 also presents the quality of life of the participants, measured with the SF-12, the WHOQOL-BREF, and the WHOQOL-OLD. Concerning the SF-12, the participants experienced a higher mental than physical quality of life. On both the WHOQOL scales, the mean scores were highest for sensory abilities and lowest for social relations (16.6 vs 14.2). For further details, refer to Table 1.

\section{Bivariate associations between sociodemographic factors and quality of life domains}

Table 2 presents the bivariate associations between the five sociodemographic factors (sex, age, marital status, education, 
Table 2 Associations between sociodemographic factors and quality of domains of the WHOQOL-BREF, the SF-I2, and the WHOQOL-OLD

\begin{tabular}{|c|c|c|c|c|c|}
\hline & Sex & Age & Marital status & Education & Income \\
\hline \multicolumn{6}{|l|}{ WHOQOL-BREF } \\
\hline Physical health & $0.09 * * *$ & $-0.06 *$ & $0.16^{* * *}$ & $0.13^{* * *}$ & $0.28 * * *$ \\
\hline Psychological & $0.06 *$ & -0.01 & $0.17^{* * *}$ & $0.15^{* * *}$ & $0.26 * * *$ \\
\hline Social relations & 0.03 & -0.03 & $0.18^{* * *}$ & 0.07 & $0.21 * * *$ \\
\hline Environmental & 0.02 & 0.02 & $0.16^{* * * *}$ & $0.25^{* * * *}$ & $0.42^{* * *}$ \\
\hline \multicolumn{6}{|l|}{ SF-12 } \\
\hline Physical & $0.12^{* * *}$ & $-0.07^{*}$ & $0.16^{* * * *}$ & $0.13 * * *$ & $0.25 * * *$ \\
\hline Mental & $0.11 * * *$ & 0.01 & $0.19 * * *$ & $0.11 * *$ & $0.24 * * *$ \\
\hline \multicolumn{6}{|l|}{ WHOQOL-OLD } \\
\hline Sensory abilities & $0.15^{* * *}$ & $-0.22 * * *$ & 0.05 & $0.10 * *$ & $0.14 * *$ \\
\hline Autonomy & $0.10^{* * * *}$ & $-0.01 * * *$ & $0.12^{* * *}$ & $0.15^{* * *}$ & $0.22 * * *$ \\
\hline Past, present, and future activities & 0.00 & 0.01 & $0.16^{* * * *}$ & $0.17^{* * *}$ & $0.34 * * *$ \\
\hline Social participation & $0.06 *$ & $-0.07 * *$ & $0.12^{* * *}$ & $0.12^{* * * *}$ & $0.20^{* * *}$ \\
\hline Death and dying & $0.11 * * *$ & -0.02 & $0.10 * *$ & 0.07 & $0.1 I^{*}$ \\
\hline Intimacy & $0.14 * * *$ & $-0.05^{*}$ & $0.50 * * *$ & $0.11 * *$ & $0.38 * * *$ \\
\hline
\end{tabular}

Notes: $* p<0.05 ; * * p<0.01 ; * * * p<0.001$.

Abbreviations: SF-12, Short-Form Health Survey; WHOQOL-BREF, World Health Organization Quality of Life Questionnaire-BREF; WHOQOL-OLD, World Health Organization Quality of Life Questionnaire-Older Adults Module.

and income) and the quality of life domains of the SF-12, the WHOQOL-BREF, and the WHOQOL-OLD, using Eta. Sex, age, marital status, education, and income were associated with 9, 6, 11, 10, and 12 quality of life domains. Then we determined per sociodemographic factor the mean scores on the 12 quality of life domains. In relation to sex, women experienced lower quality of life than men in the physical health and psychological domains of the WHOQOL-BREF, both the physical and the mental domain of the SF-12, and the death and dying and intimacy domains of the WHOQOLOLD. On the other hand, they scored significantly higher on the WHOQOL-OLD domains sensory abilities, autonomy, and social participation. Greater age was negatively correlated with the physical domain of both the WHOQOL-BREF and the SF-12 and four domains of the WHOQOL-OLD (sensory abilities, autonomy, social participation, and intimacy). Marital status (married or cohabiting) was significantly associated with all the quality of life domains, with the exception of sensory abilities $(P=0.62)$, meaning that these participants experienced higher quality of life. In addition, higher-educated participants scored higher on 10 of the 12 quality of life domains, and higher-income participants experienced better quality of life in all the quality of life domains.

\section{Regression analysis}

Table 3 presents the effects of the sociodemographic factors on the quality of life domains of the WHOQOL-BREF. The $R^{2}$ line indicates how much of the variance in the quality of life domains' scores was explained by all the predictors together. The explained variance ranged from 5\% (social relations) to $17 \%$ (environmental). The effect sizes $\left(f^{2}\right)$ were small to medium for physical health, psychological, and social relations (up to 0.15 ) and medium to large for the environmental domain of quality of life $\left(f^{2}=0.21\right) .{ }^{37}$ Of the

Table 3 Effects of sociodemographic factors on quality of life domains of the WHOQOL-BREF: regression analysis

\begin{tabular}{|l|l|l|l|l|}
\hline & Physical health & Psychological & Social relations & Environmental \\
\hline Sex (women) & -0.23 & 0.04 & $0.58^{* * *}$ & $0.26^{*}$ \\
\hline Age & $-0.03^{*}$ & 0.00 & 0.00 & 0.00 \\
\hline Marital status (married or cohabiting) & 0.22 & $0.30^{*}$ & $0.72^{* * *}$ & 0.00 \\
\hline Education & 0.02 & 0.07 & -0.01 & $0.16^{*}$ \\
\hline Income & $0.64^{* * *}$ & $0.45^{* * *}$ & $0.39^{* * *}$ & $0.83^{* * *}$ \\
\hline$R^{2}$ & $0.08^{* * *}$ & $0.07^{* * *}$ & $0.05^{* * *}$ & $0.17^{* * *}$ \\
\hline
\end{tabular}

Notes: $* P<0.05 ; * * * P<0.001$.

Abbreviation: WHOQOL-BREF, World Health Organization Quality of Life Questionnaire-BREF. 
Table 4 Effects of sociodemographic factors on quality of life domains of the SF-I2: regression analysis

\begin{tabular}{|l|l|l|}
\hline & Physical & Mental \\
\hline Sex (women) & $-4.30^{* *}$ & -1.37 \\
\hline Age & $-0.3 I^{* * *}$ & 0.04 \\
\hline Marital status (married or cohabiting) & 1.95 & $3.43^{* *}$ \\
\hline Education & 0.17 & -0.24 \\
\hline Income & $4.82^{* * *}$ & $3.27^{* * *}$ \\
\hline$R^{2}$ & $0.07^{* * *}$ & $0.06^{* * *}$ \\
\hline
\end{tabular}

Notes: $* * P<0.01$; *** $P<0.001$.

Abbreviation: SF-I2, Short-Form Health Survey.

individual sociodemographic factors, only higher income was positively associated with all the quality of life domains of the WHOQOL-BREF. Both sex and marital status were associated with two quality of life domains; age and education were only associated with one domain.

Table 4 shows the effects of the five sociodemographic factors on the two domains of the SF-12. For physical and mental quality of life, the $R^{2}$ was $6.7 \%$ and $5.8 \%$ and the corresponding effect sizes were small to medium, 0.072 and 0.061 , respectively. ${ }^{37}$ Higher income was positively associated with both domains; sex, age, and marital status were associated with one quality of life domain and education with none.

Finally, Table 5 demonstrates the effects of the sociodemographic factors on the six quality of life domains of the WHOQOL-OLD. The explained variance ranged from $1.4 \%$ (death and dying) to $26 \%$ (intimacy). The effect sizes were small to medium for death and dying, social participation, sensory abilities, autonomy, and past, present, and future activities (up to 0.15) and large for intimacy $\left(f^{2}=0.35\right) .{ }^{37}$ Being a woman was positively associated with four quality of life domains and negatively associated with one domain (death and dying). Higher income was positively associated with five of six domains; there was no association with death and dying. Married or cohabiting participants scored significantly lower on autonomy and significantly higher on social participation as well as intimacy. Greater age was negatively associated with sensory abilities and autonomy. It is striking that education did not show associations with any of the domains of the WHOQOL-OLD.

\section{Discussion}

The main aim of the present study was to compare the associations of sex, age, marital status, education, and income with quality of life assessed with the SF-12, the WHOQOL-BREF, and the WHOQOL-OLD. In addition, we aimed to determine the associations between aforementioned sociodemographic factors and quality of life. Our study was conducted in a sample of 1,492 Dutch people aged 50 years or older.

The bivariate associations reveal that the findings with regard to age, marital status, education, and income are unambiguous. Higher age was associated with lower quality of life concerning 6 of the 12 quality of life domains. Being married or cohabiting, having higher education, and having higher income were associated with higher scores on 11, 10, and all 12 quality of life domains, respectively. However, the effects of sex are quite different. Women experienced significantly lower quality of life in six quality of life domains. Conversely, they experienced significantly better quality of life in three domains, all belonging to the WHOQOL-OLD (sensory abilities, autonomy, and social participation).

The multiple linear regression analyses demonstrated that all five predictors together explained a significant part of the variance of the scores of all the quality of life domains, ranging from 5\% to $17 \%$ for the WHOQOL-BREF, $5.8 \%$ to $6.7 \%$ for the SF-12, and $1.4 \%$ to $26 \%$ for the WHOQOL-OLD. Sex (being a woman) was positively associated with six domains and negatively associated with two domains (physical in the SF-12 and death and dying in the WHOQOL-OLD), after controlling for the other four sociodemographic factors in

Table 5 Effects of sociodemographic factors on quality of life domains of the WHOQOL-OLD: regression analysis

\begin{tabular}{|l|l|l|l|l|l|l|}
\hline & $\begin{array}{l}\text { Sensory } \\
\text { abilities }\end{array}$ & Autonomy & $\begin{array}{l}\text { Past, present, and } \\
\text { future activities }\end{array}$ & $\begin{array}{l}\text { Social } \\
\text { participation }\end{array}$ & $\begin{array}{l}\text { Death and } \\
\text { dying }\end{array}$ & Intimacy \\
\hline Sex (women) & $0.73^{* * *}$ & $0.36^{* *}$ & $0.37^{* *}$ & $0.61^{* * *}$ & $-0.60^{* *}$ & 0.14 \\
\hline Age & $-0.07^{* * *}$ & $-0.03^{* * *}$ & 0.01 & -0.01 & -0.01 & -0.01 \\
\hline $\begin{array}{l}\text { Marital status (married } \\
\text { or cohabiting) }\end{array}$ & -0.20 & $-0.73^{* * *}$ & 0.14 & $0.47^{* *}$ & 0.15 & $3.00^{* * *}$ \\
\hline Education & 0.06 & 0.00 & 0.09 & 0.09 & 0.04 & 0.08 \\
\hline Income & $0.50^{* * *}$ & $0.63^{* * *}$ & $0.62^{* * *}$ & $0.42^{* * *}$ & 0.11 & $0.46^{* * *}$ \\
\hline$R^{2}$ & $0.08^{* * *}$ & $0.08^{* * *}$ & $0.10^{* * *}$ & $0.05^{* * *}$ & $0.01^{* *}$ & $0.26^{* * *}$ \\
\hline
\end{tabular}

Notes: $* * P<0.01 ; * * * P<0.001$

Abbreviation: WHOQOL-OLD, World Health Organization Quality of Life Questionnaire-Older Adults Module. 
the model. It is worth noticing that being a woman was not associated with physical health in the WHOQOL-BREF. Apparently, this is mainly caused by the difference in the operationalization of physical (health) of the WHOQOLBREF and the SF-12. The findings concerning social relations (WHOQOL-BREF) and social participation (WHOQOLOLD) point in the same direction; women rated the social domain of quality of life higher than men. This finding has been supported by many studies. Gobbens and Van Assen ${ }^{15}$ showed that social participation was higher in women than in men, after controlling for multidimensional frailty, in a sample of Dutch people aged 70 years or older. In a sample of US individuals aged 65 years or older from the National Health and Nutrition Examination Survey, women also scored higher on social functioning, although the operationalization was quite different from ours; the index contained two items: the number of close friends and how often a person attended church or religious services. ${ }^{17}$ Puts et al showed that social contact was considered to be one of the most important factors of quality of life in older adults; ${ }^{3}$ family and social activities were mentioned most by older people. ${ }^{38}$

Although previous studies on the associations between age and quality of life in older people have shown different findings, ${ }^{6,7,13,14}$ the present study has demonstrated that greater age was associated with lower quality of life. This applies, of course, to the physical domains of the quality of life scales, including sensory abilities, and to the autonomy domain of the WHOQOL-OLD. According to Gobbens and Van Assen, this operational definition of autonomy refers not only to psychological functioning but also to physical functioning and the social environment. ${ }^{35}$ In that case, the autonomy of an older person will depend to a very large extent on both his or her physical abilities and the support capabilities of the social environment. ${ }^{39,40}$ Additional statistical analyses showed that autonomy was significantly associated with physical health (WHOQOL-BREF), physical domain (SF-12), and sensory abilities (WHOQOL-OLD), represented by Pearson's coefficients of $0.52,0.43$, and 0.34 , respectively.

Participants who were married or cohabited scored lower in the quality of life domain autonomy and higher in five quality of life domains. These mainly concerned the psychological and social domains. Bilotta et al showed that living alone was associated with two domains of quality of life, namely social relationships and participation, in Italian people ( $\geq 65$ years); ${ }^{41}$ in this study, quality of life was measured with the OPQOL questionnaire. A study conducted in China also concluded that older people living alone rated a lack of social relations as a source of low satisfaction with their quality of life. ${ }^{12}$ In addition, living alone is associated with institutionalization in older persons after discharge from hospital, ${ }^{42}$ depression, ${ }^{43}$ and loneliness. ${ }^{44}$ Being married or cohabiting was also positively associated with the WHOQOL-OLD domain intimacy. Previous studies in the Netherlands and Slovakia support our findings. ${ }^{7,15}$ Intimacy, if not sexuality, is a continuing human need for most individuals, ${ }^{45} 48 \%$ of individuals above the age of 50 years did not perceive any change in the area of intimacy in their relationship over the years. ${ }^{46}$ However, intimacy is closely related to having social relations, including between partners. Because of the steady rise in life expectancy and the gender gap in longevity, the number of older persons who live alone is increasing. Specific attention needs to be paid to older women, because they tend to live longer than their husbands. ${ }^{47}$

In our study, education was associated with only one quality of life domain, the environmental domain of the WHOQOL-BREF. In other Dutch studies, higher-educated older people experienced better quality of life in more than one domain of the WHOQOL-BREF; ;,48 for example, in a longitudinal study, higher education predicted better future physical health as well as psychological and environmental quality of life. Unlike education, income was associated with eleven quality of life domains, with all $P$-values $<0.001$; income was not associated with the WHOQOL-OLD domain death and dying, including items related to anxiety about death. In a study by Mohammadpour et al, death anxiety was predicted by the perception of aging and age ${ }^{49}$ however, the latter was not found by Nouhi et al, including subjects in the same country (Iran). ${ }^{50}$ Education and income are the two common measures of socioeconomic status (SES). In addition, SES has an impact on health involving a diversity of mechanisms, such as psychosocial factors and health behaviors, for example emotional stress and social support. ${ }^{51,52}$ Previous studies have also shown that people with low SES are frailer than people with high SES. ${ }^{53,54}$ According to Kim et al, frailty acts as a mediator between SES and quality of life. ${ }^{55}$ In addition, Hoogendijk et al argue that frailty should be considered as a potential mediator of socioeconomic inequalities in adverse outcomes, for example institutionalization and death. ${ }^{54}$

Some limitations of our study should be noted. First, as mentioned in previous studies, ${ }^{9,27}$ we used a web-based questionnaire for collecting the data; this may have led to selection bias, because not everyone has access to the Internet. Another reason why there could be a significant selection bias is the fact that response was voluntary; possibly the people who completed the questionnaire were healthier and more satisfied 
than average with their quality of life. In addition, $60.3 \%$ of our sample consisted of men, while in the Dutch population in this age group ( $\geq 50$ years and older), the distribution was $47.4 \%$ men and $52.6 \%$ women, as measured on January 1 , $2010 .{ }^{56}$ So our sample could and did not represent properly the $50+$ Dutch population. Second, the cross-sectional nature of the present study does not allow us to establish causal links between the sociodemographic factors and the quality of life domains; a longitudinal study is required to determine such links. Third, the nature of the questionnaire, and the fact that it is self-administered, may have led to imprecise answers to the questions posed, particularly when the respondent was a very old person.

\section{Conclusion}

Our study has shown that the associations of sociodemographic factors and quality of life depend on the instruments used to assess quality of life in persons aged 50 years or older, taking into account the above-mentioned limitations. The contents of the instruments vary greatly, in particular between the WHOQOL-OLD on the one hand and the WHOQOLBREF and the SF-12 on the other. In addition, we have demonstrated that all the sociodemographic factors explain a significant part of all the quality of life domains involved. However, most effect sizes were small to medium; the clinical relevance of our findings will therefore be limited. There are other factors that explain a larger part of the quality of life scores in middle-aged and older persons, such as frailty ${ }^{9}$ and environmental factors. ${ }^{26}$ To make a good prediction of quality of life in this target group, such factors should be included in the prediction model. Of the five sociodemographic factors included in our study, income was the factor with by far the most significant associations with quality of life domains. Therefore, we recommend that health care and welfare professionals should focus in particular on people with a low income and carry out interventions aimed at improving their quality of life.

\section{Disclosure}

The authors report no conflicts of interest in this work.

\section{References}

1. The World Health organization quality of life assessment (WHOQOL): position paper from the World Health organization. Soc Sci Med. 1995; 41(10): 1403-1409.

2. Layte R, Sexton E, Savva G. Quality of life in older age: evidence from an Irish cohort study. J Am Geriatr Soc. 2013;61(Suppl 2):S299-S305.

3. Puts MT, Shekary N, Widdershoven G, Heldens J, Lips P, Deeg DJ. What does quality of life mean to older frail and non-frail community-dwelling adults in the Netherlands? Qual Life Res. 2007;16(2):263-277.
4. Lee TW, Ko IS, Lee KJ. Health promotion behaviors and quality of life among community-dwelling elderly in Korea: a cross-sectional survey. Int J Nurs Stud. 2006;43(3):293-300.

5. Netuveli G, Blane D. Quality of life in older ages. Br Med Bull. 2008; 85(1):113-126.

6. Paskulin L, Vianna L, Molzahn AE. Factors associated with quality of life of Brazilian older adults. Int Nurs Rev. 2009;56(1):109-115.

7. Soósová MS. Determinants of quality of life in elderly. Central Euro J Nurs Midwifery. 2016;7(3):484-493.

8. Top M, Eriş H, Kabalcıoğlu F. Quality of life (QOL) and attitudes toward aging in older adults in Şanlıurfa, Turkey. Res Aging. 2013;35(5): $533-562$.

9. Gobbens RJ, Luijkx KG, van Assen MA. Explaining quality of life of older people in the Netherlands using a multidimensional assessment of frailty. Qual Life Res. 2013;22(8):2051-2061.

10. Bilgili N, Arpacı F. Quality of life of older adults in Turkey. Arch Gerontol Geriatr. 2014;59(2):415-421.

11. Wiggins RD, Higgs PFD, Hyde M, Blane DB. Quality of life in the third age: key predictors of the CASP-19 measure. Ageing Soc. 2004; 24(05):693-708.

12. Chen Y, Hicks A, While AE. Quality of life and related factors: a questionnaire survey of older people living alone in mainland China. Qual Life Res. 2014;23(5):1593-1602.

13. Gambin G, Molzahn A, Fuhrmann AC, Morais EP, Paskulin LM. Quality of life of older adults in rural southern Brazil. Rural Remote Health. 2015;15(3):3300.

14. Borglin G, Jakobsson U, Edberg AK, Hallberg IR. Older people in Sweden with various degrees of present quality of life: their health, social support, everyday activities and sense of coherence. Health Soc Care Commun. 2006;14(2):136-146.

15. Gobbens RJJ, van Assen M. Associations between multidimensional frailty and quality of life among Dutch older people. Arch Gerontol Geriatr. 2017;73:69-76.

16. Ware J, Kosinski M, Keller SD. A 12-item short-form health survey: construction of scales and preliminary tests of reliability and validity. Med Care. 1996;34(3):220-233.

17. Baernholdt M, Hinton I, Yan G, Rose K, Mattos M. Factors associated with quality of life in older adults in the United States. Qual Life Res. 2012;21(3):527-534.

18. Hillerås PK, Jorm AF, Herlitz A, Winblad B. Life satisfaction among the very old: a survey on a cognitively intact sample aged 90 years or above. Int J Aging Hum Dev. 2001;52(1):71-90.

19. Hambleton P, Keeling S, McKenzie M. The jungle of quality of life: mapping measures and meanings for elders. Australas J Ageing. 2009; 28(1):3-6.

20. WHOQOL Group. Development of the World Health organization WHOQOL-BREF quality of life assessment. The WHOQOL group. Psychol Med. 1998;28(3):551-558.

21. Caballero FF, Miret M, Power M, et al. Validation of an instrument to evaluate quality of life in the aging population: WHOQOL-AGE. Health Qual Life Outcomes. 2013;11(1):177.

22. Paschoal SM, Filho WJ, Litvoc J. Development of elderly quality of life index-EQOLI: theoretical-conceptual framework, chosen methodology, and relevant items generation. Clinics. 2007;62(3):279-288.

23. Power M, Quinn K, Schmidt S; WHOQOL-OLD Group. Development of the WHOQOL-old module. Qual Life Res. 2005;14(10):2197-2214.

24. Bowling A. The psychometric properties of the older people's quality of life questionnaire, compared with the CASP-19 and the WHOQOLOLD. Curr Gerontol Geriatr Res. 2009;2009(3):1-12.

25. Gobbens RJJ. [Physical and mental dimensions of quality of life of frail older people]. Tijdschr Gerontol Geriatr. 2017;48(4):160-168. Dutch.

26. Gobbens RJJ, van Assen M. Associations of environmental factors with quality of life in older adults. Gerontologist. 2018;58(1):101-110.

27. Gobbens RJ, van Assen MA, Schalk MJ. The prediction of disability by self-reported physical frailty components of the Tilburg frailty indicator (TFI). Arch Gerontol Geriatr. 2014;59(2):280-287. 
28. Kalfoss MH, Low G, Molzahn AE. The suitability of the WHOQOLBREF for Canadian and Norwegian older adults. Eur J Ageing. 2008; 5(1):77-89.

29. Skevington SM, Lotfy M, O'Connell KA; WHOQOL Group. The World Health Organization's WHOQOL-BREF quality of life assessment: psychometric properties and results of the International field trial. A report from the WHOQOL group. Qual Life Res. 2004;13(2): 299-310.

30. Ware JE, Sherbourne CD. The MOS 36-item short-form health Survey (SF-36). I. conceptual framework and item selection. Med Care. 1992; 30(6):473-483.

31. Amir M, Lewin-Epstein N, Becker G, Buskila D. Psychometric properties of the SF-12 (Hebrew version) in a primary care population in Israel. Med Care. 2002;40(10):918-928.

32. Montazeri A, Vahdaninia M, Mousavi SJ, Omidvari S. The Iranian version of 12-Item short form health Survey (SF-12): factor structure, internal consistency and construct validity. BMC Public Health. 2009; 9(1):341.

33. Conrad I, Matschinger H, Riedel-Heller S, von Gottberg C, Kilian R. The psychometric properties of the German version of the WHOQOLOLD in the German population aged 60 and older. Health Qual Life Outcomes. 2014;12(1):105.

34. Halvorsrud L, Kalfoss M, Diseth A. Reliability and validity of the Norwegian WHOQOL-OLD module. Scand J Caring Sci. 2008;22(2): 292-305.

35. Gobbens RJ, van Assen MA. Psychometric properties of the Dutch WHOQOL-OLD. Health Qual Life Outcomes. 2016;14(1):103.

36. Central Committee on Research Involving Human Subjects. Does your study have to be reviewed? Available from: http://ccmo-online.nl (Home $>$ For investigators $>$ Review step plan RC $>$ WMO). Accessed June 15, 2010.

37. Cohen J. Statistical Power Analysis for the Behavioral Sciences. 2nd ed. Lawrence Erlbaum Associates, Inc.; Hillsdale, NJ: 1988.

38. Farquhar M. Elderly people's definitions of quality of life. Soc Sci Med. 1995;41(10):1439-1446.

39. Berthé A, Berthé-Sanou L, Somda S, et al. The key actors maintaining elders in functional autonomy in Bobo-Dioulasso (Burkina Faso). BMC Public Health. 2014;14(1):689.

40. Sibley A, Kersten P, Ward CD, White B, Mehta R, George S. Measuring autonomy in disabled people: validation of a new scale in a UK population. Clin Rehabil. 2006;20(9):793-803.

41. Bilotta C, Bowling A, Nicolini P, Casè A, Vergani C. Quality of life in older outpatients living alone in the community in Italy. Health Soc Care Commun. 2012;20(1):32-41.

42. Jacob L, Poletick EB. Systematic review: predictors of successful transition to community-based care for adults with chronic care needs. Care Manag J. 2008;9(4):154-165.
43. Stahl ST, Beach SR, Musa D, Schulz R. Living alone and depression: the modifying role of the perceived neighborhood environment. Aging Ment Health. 2017;21(10):1065-1071.

44. Simon MA, Chang ES, Zhang M, Ruan J, Dong X. The prevalence of loneliness among U.S. Chinese older adults. J Aging Health. 2014; 26(7):1172-1188.

45. Rheaume C, Mitty E. Sexuality and intimacy in older adults. Geriatr Nurs. 2008;29(5):342-349.

46. Kalra G, Subramanyam A, Pinto C. Sexuality: desire, activity and intimacy in the elderly. Indian J Psychiatry. 2011;53(4):300-306.

47. United Nations, Department of Economic and Social Affairs,Population Division. World population prospects. The 2017 revision. Key findings and advance tables. Working Paper No. ESA/P/WP/248. New York. 2017. Available from: https://esa.un.org/unpd/wpp/publications/Files/ WPP2017_KeyFindings.pdf. Accessed January 22, 2019.

48. Gobbens RJ, van Assen MA. The prediction of quality of life by physical, psychological and social components of frailty in communitydwelling older people. Qual Life Res. 2014;23(8):2289-2300.

49. Mohammadpour A, Sadeghmoghadam L, Shareinia H, Jahani S, Amiri F. Investigating the role of perception of aging and associated factors in death anxiety among the elderly. Clin Interv Aging. 2018;13: 405-410.

50. Nouhi E, Karimi T, Iranmanesh S. Comparing fear of death of the elderly settled in elderly's home and inhabited in city houses of Isfahan. Iran J Ageing. 2014;8(4):24-31.

51. House JS, Lepkowski JM, Kinney AM, Mero RP, Kessler RC, Herzog AR. The social stratification of aging and health. J Health Soc Behav. 1994;35(3):213-234.

52. Anderson NB, Armstead CA. Toward understanding the association of socioeconomic status and health: a new challenge for the biopsychosocial approach. Psychosom Med. 1995;57(3):213-225.

53. Mulasso A, Roppolo M, Rabaglietti E. The role of individual characteristics and physical frailty on health related quality of life (HRQOL): a cross sectional study of Italian community-dwelling older adults. Arch Gerontol Geriatr. 2014;59(3):542-548.

54. Hoogendijk EO, Heymans MW, Deeg DJH, Huisman M. Socioeconomic inequalities in frailty among older adults: results from a 10-year longitudinal study in the Netherlands. Gerontology. 2018;64(2):157-164.

55. Kim HJ, Park S, Park SH, et al. The significance of frailty in the relationship between socioeconomic status and health-related quality of life in the Korean community-dwelling elderly population: mediation analysis with bootstrapping. Qual Life Res. 2017;26(12):3323-3330.

56. Statistics Netherlands. Statline. Bevolking: geslacht, leeftijd en burgerlijke staat, 1 januari [Population: sex, age and marital status]. Available from: http://statline.cbs.nl/Statweb/publication/?DM=SLNL\&PA=746 $1 \mathrm{BEV} \& \mathrm{D} 1=0 \& \mathrm{D} 2=\mathrm{a} \& \mathrm{D} 3=106-120 \& \mathrm{D} 4=60 \& \mathrm{HDR}=\mathrm{G} 3, \mathrm{~T} \& \mathrm{STB}=\mathrm{G} 1$, G2\&VW=T. Accessed March 17, 2018.
Clinical Interventions in Aging

\section{Publish your work in this journal}

Clinical Interventions in Aging is an international, peer-reviewed journal focusing on evidence-based reports on the value or lack thereof of treatments intended to prevent or delay the onset of maladaptive correlates of aging in human beings. This journal is indexed on PubMed Central, MedLine,

\section{Dovepress}

CAS, Scopus and the Elsevier Bibliographic databases. The manuscript management system is completely online and includes a very quick and fair peer-review system, which is all easy to use. Visit http://www.dovepress. com/testimonials.php to read real quotes from published authors. 